\title{
Similarity Rules for Nonlinear Kerr-like Slab Optical Waveguides
}

\author{
Lluís Torner and Juan P. Torres
}

\begin{abstract}
We have shown that the stationary waveguiding properties of TE guided waves in a slab optical waveguide with a nonlinear Kerr-like bounding medium can be described in a compact way by means of the usual normalized effective modal index $(b)$ and a set of only four independent normalized parameters: the well-known normalized thickness $(V)$ and asymmetry measure $(a)$ of the waveguide, the generalized aspect ratio between film, and substrate refractive indexes and a new guided power measure. From an analysis starting on Buckingham's $\Pi$-theorem, we have investigated the similarity rules existing between the above waveguiding structures. Allowed and forbidden regions in the $\{b, V, a\}$-space in order that a stationary guided solution exists, have been recognized and classified, with the marginal loci separating different regions being a function of only $V$ and $a$. As a further application of the normalized parameters, we focus on the cutoff properties of the $T E_{0}$ guided wave. Both self-focusing and self-defocusing bounding media have been considered.
\end{abstract}

\section{INTRODUCTION}

$\mathrm{N}$ ONLINEAR optical waveguides exhibit a great number of interesting properties that have been extensively studied over the last years (for a review see, for example, [1]-[7]) because of their potential applications in all-optical signal processing. Switches, upper and lower threshold devices, optical limiters, nonlinear directional couplers, among other new devices, have been identified and demonstrated. The key point is the inclusion of waveguide media with intensity-dependent refractive indexes. Most of the attention has been devoted to Kerr-like materials, but more general nonlinearities [8], [9], including saturable media [10], [11], have been also analyzed.

Crucial in the design of the above devices is the determination of the waveguiding properties of the involved nonlinear waveguides. This problem is much more complicated than its linear counterpart, due basically to the following three reasons. 1) Failure of the superposition principle; 2) the fact that the solutions of the nonlinear wave equation are difficult to find and cumbersome to handle; 3 ) the number of parameters characterizing the waveguide structure is greater than in the linear case, since nonlinear coefficients of various waveguide media and the total amount of guided wave power must be specified, in

Manuscript received June 7, 1991; revised November 22, 1991.

The authors are with the Department of Signal Theory and Communications, Polytechnic University of Catalonia, 08080-Barcelona, Spain.

IEEE Log Number 9107786. addition to the linear refractive indexes, the thickness of the waveguide and the wavelength of the used radiation. In this paper we address this last point in the case of a slab waveguide in which the substrate or cladding exhibits a Kerr-like nonlinearity. Our goal is to show that the stationary waveguiding properties of such a structure can be described by means of a set of only four independent normalized variables. Scaling rules, universal results, and similarity rules between different waveguides will emerge from this analysis.

Concepts of scaling and universality, which are an extension of Fourier's [12] idea of dimension, have played an important role in most fields. They start from Buckingham's $\Pi$-theorem [13] which is the root of modern dimensional analysis and, as is well-known, in spite of being an elementary result, has an extraordinary range of applications [14]. Scaling rules in optical waveguides where analyzed first by Kogelnik and Ramaswamy. In a paper which has become classic [15], they showed that the waveguiding properties of the linear slab waveguide can be described in a very compact and elegant way by means of only three parameters: a normalized effective index $(b)$, a normalized waveguide thickness $(V)$, and an asymmetry measure of the waveguide $(a)$. This same idea was extended later to more complicated structures, such as graded-index waveguides [16]-[19], multilayer step-index structures [20] and bidimensional waveguides [21]. Similarity rules between diverging or nonparallel waveguides have also been investigated recently [22]. To our knowledge, the first attempt addressed to obtain universal results in nonlinear waveguides was made by Chelkowski and Chrostowski [23] in the case of a Kerr-like substrate or cover. Their analysis was extended by Fontaine [24] to waveguides with a nonlinear film. In this paper four normalized parameters are used: $b, V, a$, and a normalized power measure, $b_{I}$. Unfortunately $b_{I}$ contains the value of the electric field of the guided solution at the film-substrate interface. Thus, when related to the guided wave power, $b_{I}$ becomes $b$ dependent, so its usefulness for practical purposes is limited.

We avoid this important difficulty by introducing a new normalized power measure $e_{s}$ which is linear on the guided wave power and no longer depends on the other normalized variables. Moreover, $e_{s}$ relates directly to the waveguide parameters and, consequently, to the design variables. The use of $e_{s}$ instead of another $b$-dependent power 
measure requires the introduction of an additional parameter $\delta_{n}$, which is usual in linear waveguide problems. These normalized parameters enable us to identify the similarity rules existing between different waveguides and to obtain universal plots showing their stationary $\mathrm{TE}$ waveguiding properties. As we will show later on, an additional interesting feature of the above normalized parameters comes from the fact that in the case we are dealing with here, the possible values of the normalized effective index, in order that a stationary guided solution exists, define allowed and forbidden bands in the $\{b, V$, a)-space. When considering a self-focusing substrate, allowed bands subdivide themselves into three regions, corresponding to the different types of guided solutions. The point is that the marginal loci separating the different allowed from the forbidden regions are determined through only the parameters $V$ and $a$.

This paper is organized as follows. Section II is devoted to the determination of the natural scaling rules of our problem arising from $\Pi$-theorem and to the discussion of the similarity rules infered from them. In Section III we show that the stationary waveguiding properties of the structure we are analyzing can be completely described by means of the normalized variables. In Section IV we obtain and discuss the universal plots coming from the normalized eigenvalue equation. Allowed and forbidden regions in the $\{b, V, a\}$-space are identified and classified following the scheme reported by Boardman and Egan [5]. Also, the relationship between both power measures $b_{I}$ and $e_{s}$ is discussed in various cases. As a further application of the normalized parameters, at the end of Section IV we obtain the expression of the normalized cutoff power $\left(e_{s, \text { cutoff }}\right)$ in order that guided propagation occurs. In the case of a self-focusing substrate, $e_{s \text {, cutoff is a lower }}$ threshold power and depends on the parameters $V, a$, and $\delta_{n}$, but for a self-defocusing material, $e_{s, \text { cutoff }}$, which now stands for the maximum allowed guided power, is found to be a function of only $V$ and $a$. Finally, in the last section we briefly discuss the generalization of the reported procedure to other nonlinear structures and we stress our main results.

\section{II. ПI-THEOREM}

The waveguide structure we will consider here is shown in Fig. 1. It consists of a thin, optically linear, film with thickness $D$ and refractive index $n_{f}$, surrounded by a linear cover with refractive index $n_{c}$ and an intensity-dependent Kerr-like substrate with linear refractive index $n_{s}$ and nonlinear coefficient $\alpha_{s}=n_{s}^{2} \epsilon_{0} c n_{2 s}$, with $\epsilon_{0}$ and $c$ being the characteristic permittivity of free space and the speed of light in vacuo, respectively. We will consider monochromatic TE waves travelling in the $x$ direction, so the electric field has the form $\varepsilon_{y}(x, z)=(1 / 2) E_{y}(z) \exp [j(\beta x$ $-\omega t)]+$ c.c. Finally, here we will limit ourselves to the case $n_{f}>n_{s} \geq n_{c}$.

The study of the stationary waveguiding properties of the above structure requires the determination of the al-

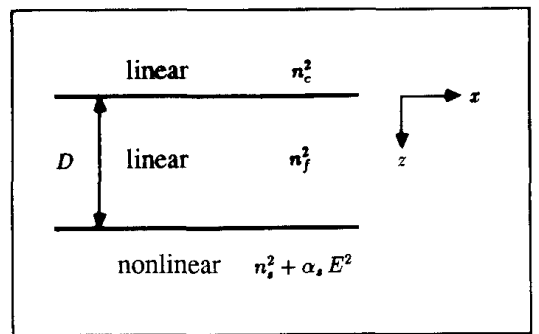

Fig. 1. Schematic structure of a slab optical waveguide with a nonlinear Kerr-like bounding medium.

lowed values of the propagation constant $\beta$ for guided solutions as a function of the waveguide parameters. In a linear waveguide these parameters are $n_{f}, n_{s}, n_{c}, D$, and the wavelength $\lambda$. Since in our case the substrate is an intensity-dependent material, the solution will also depend on the nonlinear coefficient $n_{2 s}$ and on the total guided wave power $P$. Accordingly, in this case the solution of the problem for the propagation constant can be formally expressed as

$$
N=\mathscr{N}\left(n_{f}, n_{s}, n_{c}, n_{2 s}, P, D, \lambda\right)
$$

where $N=\beta / k_{0}$ is the effective modal index, with $k_{0}$ being the free-space wavenumber. Generally speaking $\mathfrak{N}$ will be a complicated transcendental function. The point is that if we understand $\mathfrak{U}$ to be a function of the above seven variables, it contains some redundant information. This statement follows from elementary dimensional analysis.

Since the effective index is a dimensionless quantity, the well-known Buckingham П-theorem states that it must be expressed as a function of only dimensionless quantities, which are referred to as $\Pi$-monomials. It is easy to verify that the only independent dimensionless monomials which can be made with dimensional parameters appearing in (1), $\left\{n_{2 s}, P, D, \lambda\right\}$, are

$$
\Pi_{1}=k_{0} D
$$

and

$$
\Pi_{2}=\frac{n_{2 s} P}{D}
$$

Therefore, we can write

$$
N=\Re\left(n_{f}, n_{s}, n_{c}, \Pi_{1}, \Pi_{2}\right) .
$$

The monomial $\Pi_{1}$ makes clear the well-known scaling rule between $D$ and $\lambda$ which occurs in optical waveguides made in optically linear materials. On the other hand, $\Pi_{2}$ contains three parameters which play a crucial role in the nonlinear behavior of the guided waves: the nonlinear nature of the material forming the substrate, the total amount of guided power, and the thickness of the waveguide, related to the fraction of the total guided power which is carried by the nonlinear medium. Equations (2)-(4) point out how the nonlinear nature of one medium forming the waveguide causes the similarity rule stated by $\Pi_{1}$ in linear 
waveguides to break, since now for a given value of this monomial, a change in $D$ and $\lambda$ must be balanced by an identical variation on the product $n_{2 s} P$ to maintain $\Pi_{2}$, as well as $N$, at a fixed value. This means that for a given value of $\Pi_{1}$, when the waveguide thickness increases, a progressively larger guided wave power is required to obtain a prefixed value of the effective index $N$.

In fact, $\Pi_{2}$ states a new similarity rule between nonlinear Kerr-like waveguides. For instance, it is obvious from (3) that as far as the effective index $N$ is concerned, a change in the nonlinear coefficient $n_{2 s}$ is exactly counterbalanced by the inverse variation in the guided power $P$. Then, we conclude that regarding the effective indexes of guided solutions, monomial $\Pi_{2}$ establishes an equivalence relation between similar waveguides with different characteristic parameters but equal $\Pi_{2}$. Notice that this is true for the waveguides in which the substrate or cladding is a nonlinear material, but also for those in which the nonlinear medium constitutes the film.

The monomials $\Pi_{1}$ and $\Pi_{2}$ point out the natural scaling rules of our system, and therefore, should be retained in the definition of the variables describing it.

\section{Formal APPROACH}

$\Pi$-theorem does not inform us as to how the refractive indexes $n_{f}, n_{s}$, and $n_{c}$ intermix with $\Pi_{1}$ and $\Pi_{2}$ in the arguments of the function $\mathfrak{T}$. To investigate this point we will only remember that the function $\mathfrak{N}$ can be obtained from the resolution of the wave equation with the required boundary conditions at the dielectric interfaces. In the case we are dealing with here, the wave equation reads

$$
\begin{gathered}
\left\{\partial_{z}^{2}+k_{0}^{2}\left[n_{c}^{2}-N^{2}\right]\right\} E_{y}(z)=0, \quad z<0 \\
\left\{\partial_{z}^{2}+k_{0}^{2}\left[n_{f}^{2}-N^{2}\right]\right\} E_{y}(z)=0, \quad 0<z<D \\
\left\{\partial_{z}^{2}+k_{0}^{2}\left[n_{s}^{2}-N^{2}+\alpha_{s} E_{y}^{2}(z)\right]\right\} E_{y}(z)=0, \quad D<z
\end{gathered}
$$

and boundary conditions require the continuity of $E_{y}(z)$ and $\partial_{z} E_{y}(z)$ at $z=0$ and $z=D$. Because of the nonlinear nature of the material forming the substrate, these equations are not enough to completely describe our problem and so, we must also specify the total amount of guided wave power

$$
P=\frac{1}{2} N \epsilon_{0} c \int_{-\infty}^{\infty} E_{y}^{2}(z) d z .
$$

We will rewrite these expressions using the dimensionless coordinate $\xi \equiv z / D$ and the dimensionless electric field function $\Psi(\xi) \equiv E_{y}(\xi) / E_{0}$, with $E_{0}$ being a constant which will be specified later on. In terms of these new variables and keeping in mind the case of the linear waveguide, (5)-(7) become

$$
\begin{aligned}
\left\{\partial_{\xi}^{2}-V^{2}[a+b]\right\} \Psi(\xi) & =0, & & \xi<0 \\
\left\{\partial_{\xi}^{2}+V^{2}[1-b]\right\} \Psi(\xi) & =0, & & 0<\xi<1 \\
\left\{\partial_{\xi}^{2}-V^{2} b+e_{s} \Psi^{2}(\xi)\right\} \Psi(\xi) & =0, & & 1<\xi .
\end{aligned}
$$

Moreover, now the boundary conditions require the continuity of $\Psi(\xi)$ and $\partial_{\xi} \Psi(\xi)$ at $\xi=0$ and $\xi=1$. In these expressions we have made use of the usual definitions of the normalized effective index [15]

$$
b=\frac{N^{2}-n_{s}^{2}}{n_{f}^{2}-n_{s}^{2}}
$$

the normalized thickness

$$
V=\Pi_{1} \sqrt{n_{f}^{2}-n_{s}^{2}}
$$

and the asymmetry measure

$$
a=\frac{n_{s}^{2}-n_{c}^{2}}{n_{f}^{2}-n_{s}^{2}} .
$$

Finally, the parameter $e_{s}$ is defined as

$$
e_{s}=\Pi_{1}^{2} \alpha_{s} E_{0}^{2} \text {. }
$$

The meaning of this new parameter depends on the definition of the constant $E_{0}$. This is a somewhat arbitrary but also crucial choice, since it states the actual usefulness of $e_{s}$. If one takes $E_{0} \equiv E_{y}(z=D), e_{s}$ becomes proportional to the parameter $b_{I}$ used by Chelkowski and Chrostowski but then, when related to the guided wave power, it becomes $b$ dependent. Instead of this, we have taken a definition based on the following three requirements. First, we must take into account that $e_{s}$ should be a power measure and, if it is possible, a simple one. Second, the definition of $e_{s}$ must allow us to rewrite (8) for the guided wave power in terms of only dimensionless parameters. Finally, $e_{s}$ must be an independent parameter, i.e., not depending on the normalized effective index $b$. Accordingly, we have taken the definition

$$
E_{0}^{2} \equiv \frac{2 P}{n_{s} \epsilon_{0} c D} .
$$

Substituting this expression into (15) one arrives at

$$
e_{s}=2 n_{s} \Pi_{1}^{2} \Pi_{2} \text {. }
$$

This expresssion shows that the parameter $e_{s}$ is a power measure. Moreover, according to the definition of $\Pi_{2}, e_{s}$ depends linearly on the power $P$. It is also worth noticing that the parameter $e_{s}$ given by (17) is proportional to the power measure used by Boardman and Egan [5], $\left(P / P_{0}\right)_{B-E}=P n_{2 s} n_{s}^{2} k_{0} / 2$, among other authors [8]. In fact, one has

$$
e_{s}=\frac{4 \Pi_{1}}{n_{s}}\left(\frac{P}{P_{0}}\right)_{B-E} .
$$

On the other hand, rearranging (8) in terms of $\xi$ and $\Psi(\xi)$ and using (16), one arrives at

$$
\int_{-\infty}^{\infty} \Psi^{2}(\xi) d \xi=\frac{1}{\sqrt{1+\left(\delta_{n}^{2}-1\right) b}}
$$

where

$$
\delta_{n}=\frac{n_{f}}{n_{s}}
$$


Then, in view of (9), (10), (11), (19), and the boundary conditions at $\xi=0,1$, we conclude that

$$
b=\Re_{s}\left(V, a, e_{s}, \delta_{n}\right) \text {. }
$$

The ratio $\delta_{n}$ is an usual parameter in linear waveguide problems, since it appears in the analysis of the TM modes of the slab waveguide and it is used also as a generalized aspect ratio in bidimensional geometries. However, in our case, $\delta_{n}$ enters the problem due to the nonlinear nature of the material forming the substrate. When the power measure $e_{s}$ vanishes, the nonlinear term in (11) disappears and thus (19) becomes unnecessary to specify the whole differential problem defined by (9)-(11). Therefore, in this case the $\delta_{n}$ dependence of $\mathcal{B}_{s}$ disappears also and (21) reduces to the function $b_{L}=\widehat{B}_{L}(V, a)$ given by

$$
V \sqrt{1-b_{L}}=m \pi+\tan ^{-1} \sqrt{\frac{b_{L}}{1-b_{L}}}+\tan ^{-1} \sqrt{\frac{a+b_{L}}{1-b_{L}}}
$$

with $b_{L}$ being the normalized effective index of the linear or low-power waveguide. In most practical applications the difference between the film and substrate refractive indexes amount to a small value, so typically one has $\left(\delta_{n}^{2}-1\right)<<1$. Also, we can assume that for small guided wave powers, $b<1$. Therefore in these conditions, (19) becomes weakly $\delta_{n}$ dependent and, accordingly, one can take $b \simeq \mathbb{B}_{s}\left(V, a, e_{s}\right)$. On the contrary, we expect that the dependence of the function $B_{s}$ on the parameter $\delta_{n}$ will increase as the power measure $e_{s}$ grows.

On the other hand, according to (13), (14), (17), and (20) we can take every one of the four parameters $V, a$, $e_{s}$, and $\delta_{n}$ as independent of each other. Moreover, in the case we are considering here $\left(n_{f}>n_{s} \geq n_{c}\right)$, the normalized variables $V, a$, and $e_{s}$ can take any positive value. On the contrary, for a given, positive value of the asymmetry measure there is a maximum allowed value of the parameter $\delta_{n}$. From (14) it is found to be

$$
\delta_{n, \max }=\sqrt{1+\frac{1}{a}}
$$

and $1<\delta_{n}<\delta_{n, \max }$. When $a \sim 0$, one has $\delta_{n, \max } \gg 1$, and thus the possible values of $\delta_{n}$ are not restricted in practice. However in the case of asymmetric waveguides $\delta_{n, \max } \sim 1$, and thus the existence of this maximum value has deep implications since one has $\left(\delta_{n}^{2}-1\right)<\left(\delta_{n, \max }^{2}-\right.$ 1) $=1 / a$. Therefore when $a \gg 1$, the term containing the parameter $\delta_{n}$ in (19) amounts to a negligible value unless $b \sim a$. In these conditions the dependence of the function $B_{s}$ on $\delta_{n}$ can be neglected and one gets $b \simeq$ $\mathrm{B}_{s}\left(V, a, e_{s}\right)$, as above. Clearly, this will be no longer true for guided solutions having $b \gg 1$.

Finally, we wish to emphasize that the relationship between the parameter $e_{s}$ and $P$ stated through (16) is crucial. Without it, the meaning of the definition (15) would not be clear in some cases, to such an extent that some numerical values of the parameter $e_{s}$ which would be taken as reasonable from (15), in fact cannot be reached in practice with a finite guided power. In contrast, (17) contains essentially the monomials $\Pi_{1}$ and $\Pi_{2}$, and thus the numerical values of $e_{s}$ can be directly related to the actual characteristic parameters of the waveguide and, consequently, to the design variables.

\section{Discussion}

\section{A. Normalized Eigenvalue Equation}

In this section we will deal with the universal plots for the guiding properties of the waveguide in Fig. 1 obtained by means of the above introduced normalized parameters.

The solution of the differential equations (9)-(11) is now well-known [1]-[7], [25], [26]. For a self-focusing substrate $\left(n_{2 s}>0\right.$ or $\left.\Pi_{2}>0\right)$, in terms of the normalized variables, one has

$$
\begin{aligned}
& \Psi_{c}(\xi)=\frac{\Psi_{0}}{\sqrt{e_{s}}} \exp [V \sqrt{a+b} \xi], \quad \xi \leq 0 \\
& \Psi_{f}(\xi)=\frac{\Psi_{0}}{\sqrt{e_{s}}}[\cos (V \sqrt{1-b} \xi)+A \sin (V \sqrt{1-b} \xi)] \\
& 0 \leq \xi \leq 1 \\
& \Psi_{s}(\xi)=\sqrt{\frac{2 b}{e_{s}}} \frac{V}{\cosh \left(V \sqrt{b}\left(\xi+\xi_{0}-1\right)\right)} \quad 1 \leq \xi
\end{aligned}
$$

where use has been made of the definition $A \equiv$ $\sqrt{(a+b) /(1-b)}$. The constants $\Psi_{0}$ and $\xi_{0}$ follow from the boundary conditions at $\xi=1$, and are obtained, respectively, through the expressions

$$
\begin{aligned}
\Psi_{0}= & \sqrt{2 b} \frac{V}{\cosh \left(V \sqrt{b} \xi_{0}\right)} \\
& \cdot \frac{1}{\cos (V \sqrt{1-b})+A \sin (V \sqrt{1-b})}
\end{aligned}
$$

$\tanh \left(V \sqrt{b} \xi_{0}\right)$

$$
=\sqrt{\frac{1-b}{b}} \frac{\sin (V \sqrt{1-b})-A \cos (V \sqrt{1-b})}{\cos (V \sqrt{1-b})+A \sin (V \sqrt{1-b})} .
$$

The substitution of (24)-(26) into (19) yields the expression for the guided wave power in terms of $e_{s}$. One arrives at

$$
e_{s}=\sqrt{1+\left(\delta_{n}^{2}-1\right) b}\left\{I_{c}+I_{f}+I_{s}\right\}
$$

with

$$
I_{c}=\frac{\Psi_{0}^{2}}{2 V \sqrt{a+b}}
$$




$$
\begin{aligned}
I_{f}= & \Psi_{0}^{2}\left[\frac{1+A^{2}}{2}+\frac{1-A^{2}}{2} \frac{\sin (2 V \sqrt{1-b})}{2 V \sqrt{1-b}}\right. \\
& \left.+A \frac{\sin ^{2}(V \sqrt{1-b})}{V \sqrt{1-b}}\right] \\
I_{s}= & 2 V \sqrt{b}\left[1-\tanh \left(V \sqrt{b} \xi_{0}\right)\right] .
\end{aligned}
$$

The same expressions hold for a self-defocusing substrate $\left(n_{2 s}<0\right.$ or $\left.\Pi_{2}<0\right)$, provided that $e_{s}$ is replaced everywhere by $\left|e_{s}\right|$, each of the hyperbolic functions cosh or sinh is replaced by sinh or cosh, respectively, in (26), (27), (28), and (32), and $I_{s}$ is replaced by $-I_{s}$ in (32). Further attention must be devoted to the expression equivalent to (28), which accordingly with the above rule now writes

$$
\begin{aligned}
\tanh \left(V \sqrt{b} \xi_{0}\right) \\
=\sqrt{\frac{b}{1-b}} \frac{\cos (V \sqrt{1-b})+A \sin (V \sqrt{1-b})}{\sin (V \sqrt{1-b})-A \cos (V \sqrt{1-b})}
\end{aligned}
$$

since, in this case, we must also take into account the condition $\xi_{0}>0$, to ensure the right behavior of $\Psi_{s}(\xi)$ everywhere at $\xi>1$. Finally, in the case of a self-focusing medium, (24)-(32) hold for both complementary cases $b \leq 1$ and $b>1$, provided that the properties of the trigonometric functions for pure imaginary arguments are used.

\section{B. Allowed Regions in the $b-V$ Plane}

Equation (29) constitutes the implicit function $b=$ $\beta_{s}\left(V, a, e_{s}, \delta_{n}\right)$ defined in $(21)$; therefore it is the normalized eigenvalue equation. However, when we solve it to obtain the allowed normalized effective indexes $b$ for the guided solutions, we must always verify that the obtained values satisfy the conditions (28) or (33), in the case of a self-focusing or a self-defocusing substrate, respectively. Then, since the hyperbolic tangent function appearing in the left-hand side of these expressions verifies $-1<\tanh \left(V \sqrt{b} \xi_{0}\right)<1$, for $V \sqrt{b} \xi_{0} \in(-\infty, \infty)$, we conclude that there are some forbidden values of the normalized effective index $b$ which make the right-hand side of (28) or (33) fall outside this range. The point is that the locus in the $\left\{b, V, a, e_{s}, \delta_{n}\right\}$-space separating allowed from forbidden regions is determined only through $b, V$, and $a$, since it comes simply from the limiting cases tanh $\left(V \sqrt{b} \xi_{0}\right) \rightarrow \pm 1$, and the RHS of (28) and (33), where these limits must be calculated, are functions of only these three parameters.

As usual in the linear regime we will draw universal plots for the normalized effective index by plotting $b$ as a function of $V$, for different values of $a, e_{s}$, and $\delta_{n}$. Then, in such charts the regions of allowed and forbidden values of $b$ in order that a stationary guided solution exists, will be referred to as the allowed and forbidden regions in the
$b-V$ plane, respectively, for each value of the asymmetry measure $a$. The marginal loci separating both regions come easily from (28) and (33). For a self-focusing substrate, the condition that the RHS of (28) be equal to \pm 1 yields

$$
V \sqrt{1-b_{c}}=m \pi+\tan ^{-1} \sqrt{\frac{a+b_{c}}{1-b_{c}}} \pm \tan ^{-1} \sqrt{\frac{b_{c}}{1-b_{c}}}
$$

for $b_{c} \leq 1$. The upper sign corresponds to the positive limit $(+1)$ and the lower to the negative one $(-1)$, with $b_{c}$ standing for the critical value at which (34) holds. For given $V$ and $a$, these values will be referred to as $b_{c+}$ and $b_{c-}$, according to the corresponding sign which has been taken in (34). Notice that for the positive sign, (34) is nothing but the normalized eigenvalue equation for the linear or low-power waveguide given by (22). In Fig. 2 the set of allowed regions defined by (34) are shown for an asymmetric waveguide.

The allowed regions in the $b-V$ plane contain the three possible types of guided waves. These types of guided solutions differentiate from each other by the shape of the corresponding electric field function. They are [5]:

1) Trigonometric functions in the film; purely exponential decay in the substrate. The maximum of the electric field at the substrate occurs at $\xi=1$. This is a pure guided wave solution.

2) Trigonometric functions in the film; bulged exponential decay in the substrate. The maximum of the electric field at the substrate takes place at $\xi>1$. This is a bulged guided wave solution.

3) Hyperbolic functions in the film. The maximum of the electric field occurs at the substrate. This is a surface wave solution.

The surface waves occur above the line $b=1$ in the $b-V$ charts. On the other hand, the locus in the $b-V$ plane separating pure from bulged guided wave solutions come again from (28). In view of (26), we realize that if $\xi_{0}>$ 0 , the field solution at the substrate, $\Psi_{s}(\xi)$, decays exponentially from $\xi=1$ and thus corresponds to a pure guided wave solution. On the contrary, when $\xi_{0}<0$, $\Psi_{s}(\xi)$ reaches its maximum value at $\xi=1-\xi_{0}>1$, and therefore corresponds to a bulged guided wave. We conclude that the marginal loci in the $b-V$ plane separating both types of guided waves are obtained from the condition $\xi_{0}=0$. Taking this value in (28) one arrives at

$$
V \sqrt{1-b_{c 0}}=m \pi+\tan ^{-1} \sqrt{\frac{a+b_{c 0}}{1-b_{c 0}}} .
$$

Consistently with the definition of $b_{c+}$ and $b_{c-}$, the values of $b$ which verify this equation for given $V$ and $a$ are denoted as $b_{c 0}$. The curve $b_{c 0}(V)$ has also been plotted in Fig. 2.

For an asymmetric waveguide, Fig. 2 shows that when the normalized thickness is greater than the low-power 


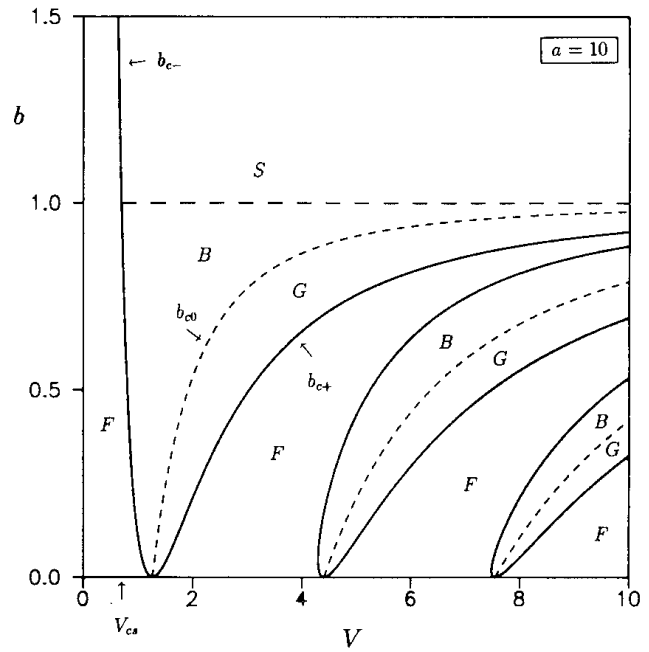

Fig. 2. Allowed regions in the $b-V$ chart for TE guided propagation in an asymmetric waveguide $(a=10)$ with a self-focusing bounding medium. Labels $\mathbf{F}$ indicate forbidden regions whereas $\mathbf{G}, \mathbf{B}$, and $\mathbf{S}$ correspond to allowed regions for pure guided waves, bulged guided waves, and surface waves, respectively. The marginal curves $b_{c+}(V), b_{c-}(V)$, and $b_{c 0}(V)$ separating different regions start at $V=V_{L \text {, cutuff. The characteristic normalized }}$ thickness $V_{c r}$ discussed in the text has been also indicated.

cutoff thickness $\left(V_{L, \text { cutoff }}=m \pi+\tan ^{-1} \sqrt{a}\right)$, the three types of $\mathrm{TE}_{0}$ guided solutions are possible, the actual occurrence of one of them depending on the values of $e_{s}$ and $\delta_{n}$, and on the excitation conditions. However, when $V<$ $V_{L \text {, cutoff }}$ the pure guided wave solutions become no longer possible. In addition, there is still another important characteristic normalized thickness, $V_{c s}$. Below it, only surface waves are allowed in the nonlinear waveguide. The

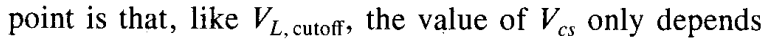
on the asymmetry measure of the waveguide. According to Fig. $2, V_{c s}$ is obtained by taking the limit $b_{c-} \rightarrow 1$ in (34). One simply gets

$$
V_{c s}=1-\frac{1}{\sqrt{1+a}} .
$$

When $a \rightarrow 0$, one also has $V_{c s} \rightarrow 0$, whereas for strongly asymmetric waveguides, $V_{c s}$ reaches its maximum value: $V_{c s}(a>1) \rightarrow 1$.

Contrary to the former case, in a symmetric waveguide $(a=0)$ the low-power normalized cutoff thickness vanishes, i.e., $V_{L \text {, cutoff }}=0$. Therefore in such a waveguide, the three types of $\mathrm{TE}_{0}$ guided solutions become allowed for any value of the normalized thickness.

We will look now at a self-defocusing substrate. In this case the condition $\tanh \left(V \sqrt{b} \xi_{0}\right) \rightarrow-1$ makes no sense, since as we have already mentioned, in this case $\xi_{0}$ must take positive values. Therefore, now the marginal loci separating allowed and forbidden regions in the $b-V$ plane are defined by the conditions tanh $\left(V \sqrt{b_{c}} \xi_{0}\right) \rightarrow 1$ and tanh $\left(V \sqrt{b_{c}} \xi_{0}\right) \rightarrow 0$. Using (33), the first limit leads back to (34) for $b_{c+}$, whereas the second

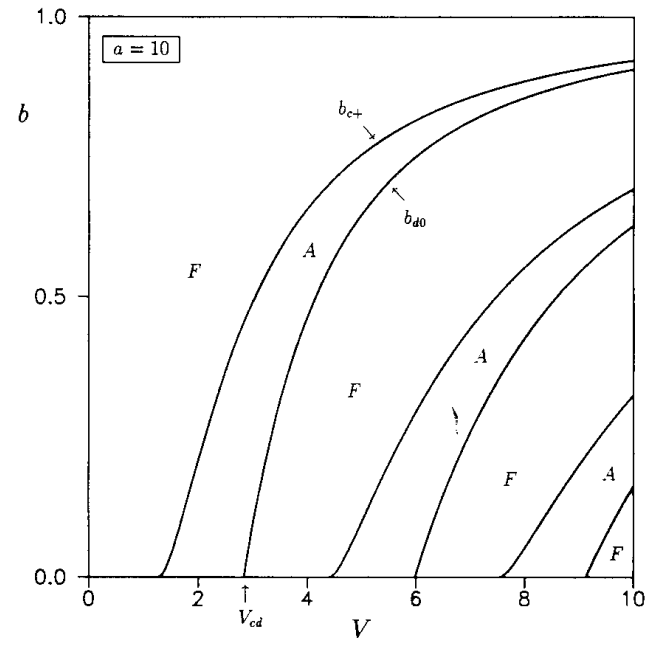

Fig. 3. Allowed (A) and forbidden $(\mathbf{F})$ regions in the $b-V$ chart for $\mathrm{TE}$ guided propagation in an asymmetric waveguide with a self-defocusing bounding medium. The characteristic normalized thickness $V_{c d}$ has only been indicated for the $\mathrm{TE}_{0}$-band.

one yields $b_{c}=0$, for $V_{c d} \geq V \geq V_{L, \text { cutoff }}$, and

$$
V \sqrt{1-b_{d 0}}=(m+1) \pi-\tan ^{-1} \sqrt{\frac{1-b_{d 0}}{a+b_{d 0}}}
$$

for $V \geq V_{c d}$, with $b_{d 0}$ being defined by this equation. Here $V_{c d}$ stands for the new characteristic normalized thickness

$$
V_{c d}=(m+1) \pi-\tan ^{-1} \frac{1}{\sqrt{a}} .
$$

Below $V_{c d}$, the minimum allowed value of the normalized effective index to ensure that the solutions be guided is $b$ $=0$, whereas when $V \geq V_{c d}$, one has $b_{c+}>b>b_{d 0}$. The corresponding allowed regions in the $b-V$ chart are shown in Fig. 3.

Figs. 2 and 3 deserve an additional comment. According to the plots we must emphasize that the allowed regions in the $b-V$ plane corresponding to TE guided solutions of different order do not overlap. This statement holds for both self-focusing and self-defocusing media and implies that for a fixed value of the asymmetry measure of the waveguide, an arbitrary, allowed pair of values of $V$ and $b$ can only occur for a single $\mathrm{TE}_{m}$ guided wave.

In Figs. 4-7 we have plotted the normalized effective index of the lowest order TE guided wave as a function of the normalized thickness for different values of the power measure $e_{s}$. Figs. 4 and 5 correspond to a self-focusing medium whereas Figs. 6 and 7 to a self-defocusing case. To illustrate the effects due to a nonvanishing asymmetry measure, a perfectly symmetric waveguide $(a=0)$ and an asymmetric one ( $a=10$ ) have been considered. The plots show how, starting from the curve $b_{c+}(V)$, the values of $b$ as a function of $V$ go over all the allowed region as the power measure increases from $e_{s}=0$. However there is a crucial difference between symmetric and 


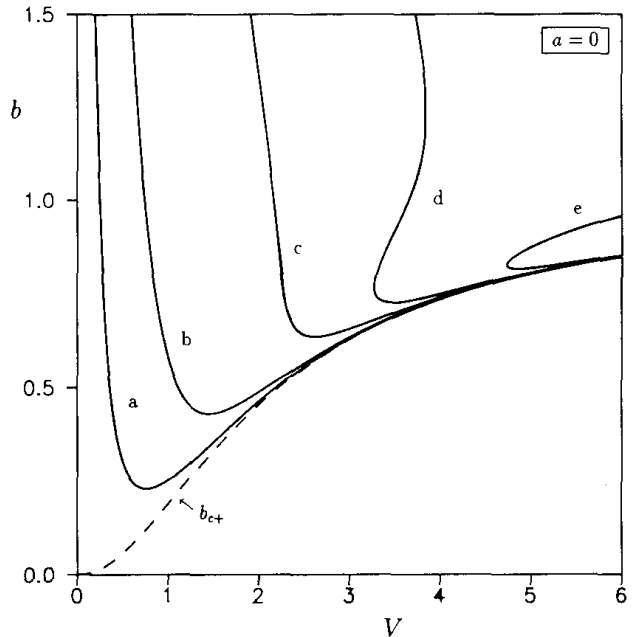

Fig. 4. Normalized effective index of the $\mathrm{TE}_{0}$ guided wave as a function of the normalized thickness in a perfectly symmetric waveguide with a selffocusing substrate, for different values of the power measure. Curves labeled $a, b, c, d$, and $e$ correspond to $e_{5}=1,3,10,20$, and 50, respectively. In all cases $\delta_{b}=1.013$. The curve $b_{c+}(V)$ corresponding to the linear or low-power limit has been plotted with a dashed line. There is not cutoff for the $\mathrm{TE}_{0}$ guided wave.

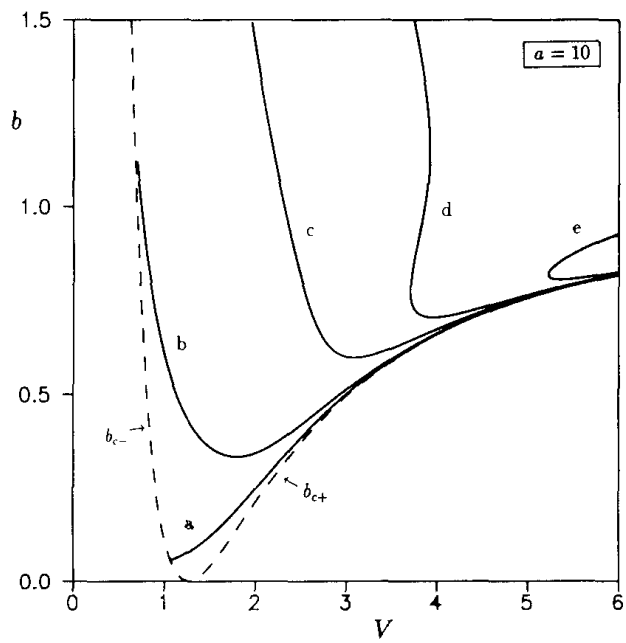

Fig. 5. Same as in Fig. 4 but for an asymmetric waveguide. Dashed lines correspond to the curves $b_{c+}(V)$ and $b_{c-}(V)$. Contrary to the symmetric case, below $V_{L \text { cutoff }}$ there is a lower threshold value of $e_{s}$ in order that guided propagation be allowed. Cutoff occurs at $b=b_{c-}$.

asymmetric waveguides [1]-[7]. According to Figs. 4 and 6 in a perfectly symmetric waveguide there is not cutoff for the $\mathrm{TE}_{0}$ guided wave. On the contrary, Figs. 5 and 7 show that this is no longer true in the case of asymmetric structures. We will deal with this important fact in next section.

Fig. 8 illustrates the dependence of the normalized effective index of the $T E_{0}$ guided solution on the parameter $\delta_{n}$, in an asymmetric waveguide with a self-focusing substrate. As expected (since $\delta_{n, \max }=\sqrt{2}$ ), away from the cutoff point, the normalized effective index depends very

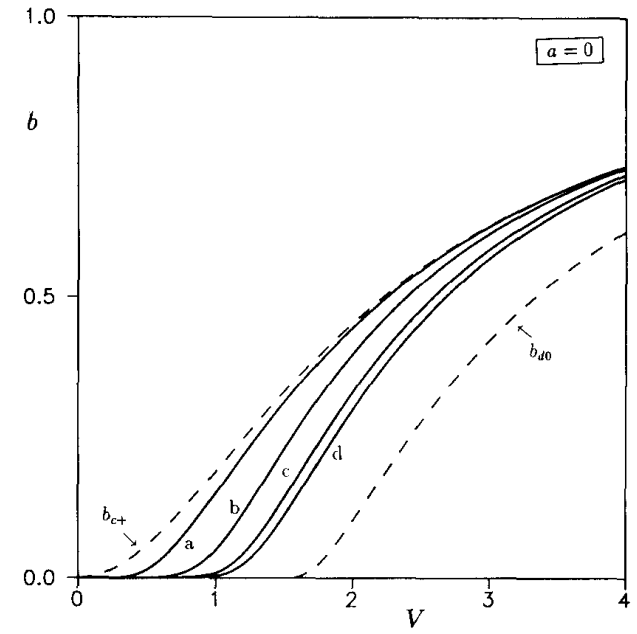

Fig. 6. Normalized effective index of the $\mathrm{TE}_{0}$ guided wave as a function of the normalized thickness in a perfectly symmetric waveguide with a selfdefocusing substrate. Labels $a, b, c$, and $d$ correspond to $\left|e_{s}\right|=1,10,50$, and 100 , respectively, and here also $\delta_{n}=1.013$. Dashes correspond to the curves $b_{c+}(V)$ and $b_{d 0}(V)$.

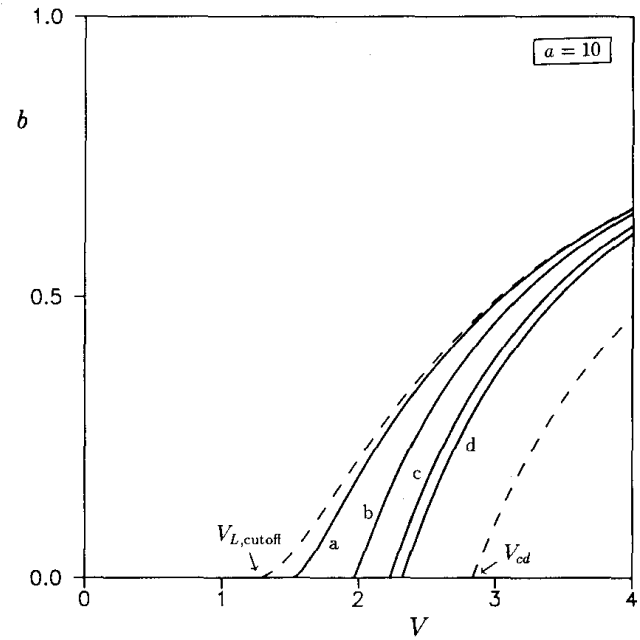

Fig. 7. Same as in Fig. 6 but for an asymmetric waveguide. In the range $V_{c d}>V>V_{L, \text { cutoff, }}$, cutoff occurs at $b=0$. Above $V_{c d}$ there is not cutoff.

smoothly on $\delta_{n}$. Also, from the plot we verify that when $\delta_{n}$ increases, $b$ approaches its linear value $b_{L}$. This is so because, for a given value of the power measure, the relative importance of the self-focusing effect at the substrate becomes lower as the ratio $n_{f} / n_{s}$ increases. Finally, note that the different values of $\delta_{n}$ in Fig. 8 render very similar values of the normalized effective index in the

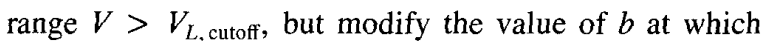
the nonlinear guided wave reaches its cutoff. We will return to this question in Fig. 12.

To end this section, in Figs. 9 and 10 we have analyzed the relationship between the $b$ dependent power measure $b_{I}$ and $e_{s}$, in some typical cases. As expected, since $e_{s}$ is a linear power measure, $b_{I}$ depends linearly on $e_{s}$ as well 


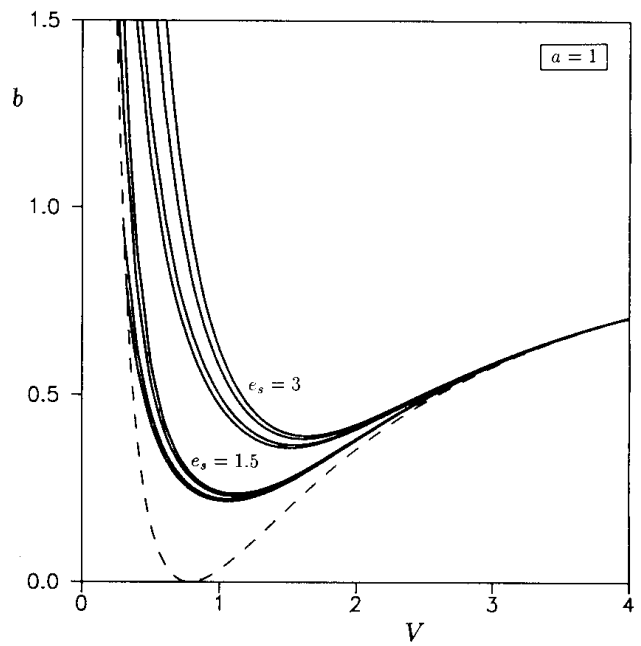

Fig. 8. This plot illustrates the dependence of the normalized effective index of the $\mathrm{TE}_{0}$ guided wave on the parameter $\delta_{n}$, in the case of an asymmetric waveguide with a self-focusing bounding medium, for two different values of the power measure $\left(e_{s}=1.5\right.$ and $\left.e_{s}=3.0\right)$. The four curves drawn for each value of $e_{s}$ correspond, from left to right, to $\delta_{n}=1.01$, $1.1,1.3$, and 1.4 .

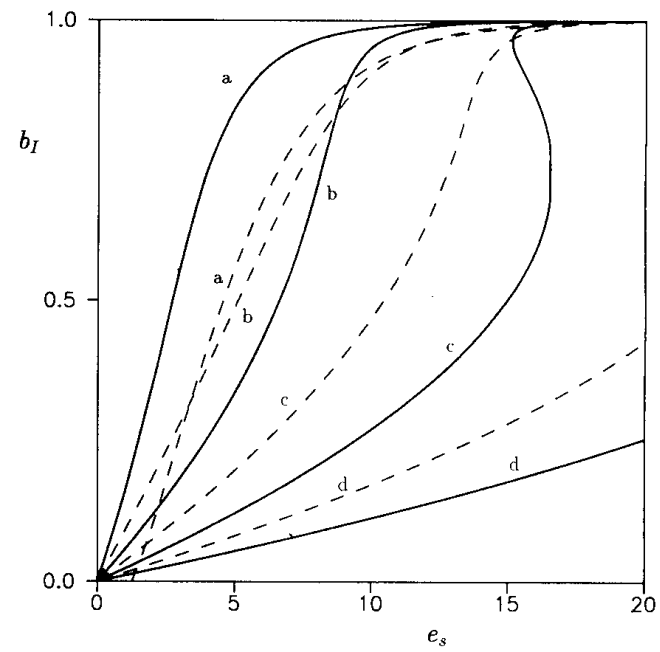

Fig. 9. The $b$-dependent power measure $b_{I}$ is plotted versus $e_{\text {, for the }} \mathrm{TE}_{0}$ guided wave in a waveguide with a self-focusing substrate, for different values of the normalized thickness. Continuous line: perfectly symmetric waveguide $(a=0)$; dashed line: asymmetric waveguide with $a=10$. Labels $a, b, c$, and $d$ stand for $V=1,2,3$, and 4 , respectively, and in all cases $\delta_{n}=1.013$

for small values of $e_{s}$. However, this becomes no longer true out of this range. In Fig. 9 the curve corresponding to the asymmetric waveguide with $V=1$ does not start at $e_{s}=0$ since this normalized thickness is less than $V_{L \text {, cutoff }}$ and thus for low powers, the $\mathrm{TE}_{0}$ guided wave is cutoff. Likewise, in Fig. 10 the curve corresponding to $V=1$ and $a=10$ does not appear since in the self-defocusing case, the $\mathrm{TE}_{0}$ guided wave is always cutoff for this normalized thickness. Fig. 10 shows also that, for given typical values of $V, a$, and $\delta_{n}$, great values of $b_{I}$ can only be reached with enormous $e_{s}$.

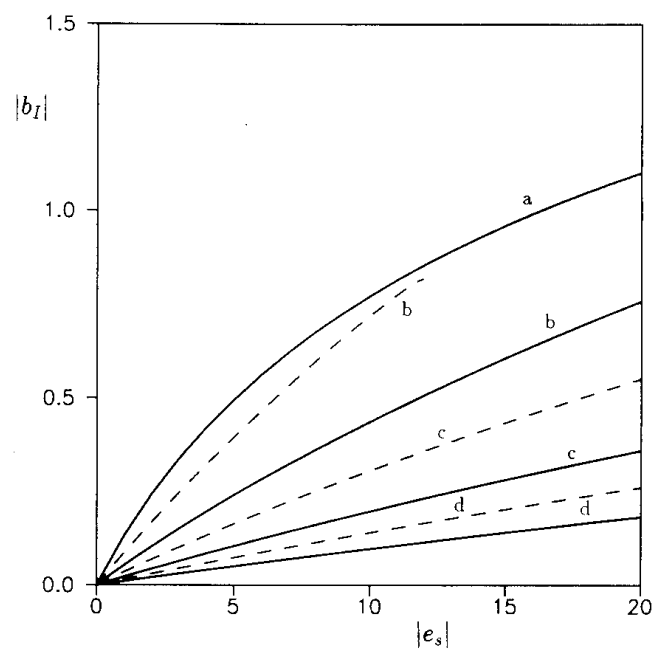

Fig. 10. Same as in Fig. 9 but for a self-defocusing bounding medium.

\section{Cutoff Power}

As has been pointed out by numerous authors [1]-[7], one of the most interesting features of the optical waveguides with a nonlinear substrate is that they can act as power limiters. For a self-focusing material this possibility arises from the fact that if the waveguide thickness is less than the low-power cutoff thickness, there is a minimum value of the guided wave power in order that a guided solution be allowed. This behavior can be clearly seen in Figs. 5 and 8. On the other hand, for a self-defocusing material, there is an upper value of the guided power to allow a guided solution, as can be seen in Figs. 6 and 7. Both these critical powers can be easily calculated and expressed in a very compact way be means of the normalized parameters. We will limit ourselves to the $\mathrm{TE}_{0}$ guided wave.

We will start with a self-focusing substrate. In this case, according to our discussion in Section IV-B, below the low-power normalized cutoff thickness, the minimum value of $b$ for given $V$ and $a$ is $b_{c-}$. Then, by taking the limit $b \rightarrow b_{c-}$ in the eigenvalue equation (29), we obtain the lower threshold $e_{s}$ value for guided propagation to occur. One arrives at

$$
e_{s, \text { cutoff }}=4 V \sqrt{b_{c-}} \sqrt{1+\left(\delta_{n}^{2}-1\right) b_{c-}}
$$

which $b_{c-}$ coming from (34). As mentioned above, this expression holds only for $V<\tan ^{-1} \sqrt{a}$. In the neighborhood of $V_{L \text {, cutoff }}$, one has $b_{c-}<<1$; thus, since in most cases it is also true that $\left(\delta_{n}^{2}-1\right)<<1$ (for instance, in strongly asymmetric waveguides one has $\delta_{n, \max } \sim 1$ and thus this condition is always fulfilled), we can write with a high degree of approximation $e_{s, \text { cutoff }} \simeq 4 V \sqrt{b_{c-}}$, for values of $V$ in this range. Since $b_{c}$ - only depends on $V$ and $a$, we conclude that this last expression is also a function of only these two parameters.

The behavior of $e_{s \text {, cutoff }}$ as a function of $V$ for different values of $a$ and $\delta_{n}$ is shown in Figs. 11 and 12, respec- 


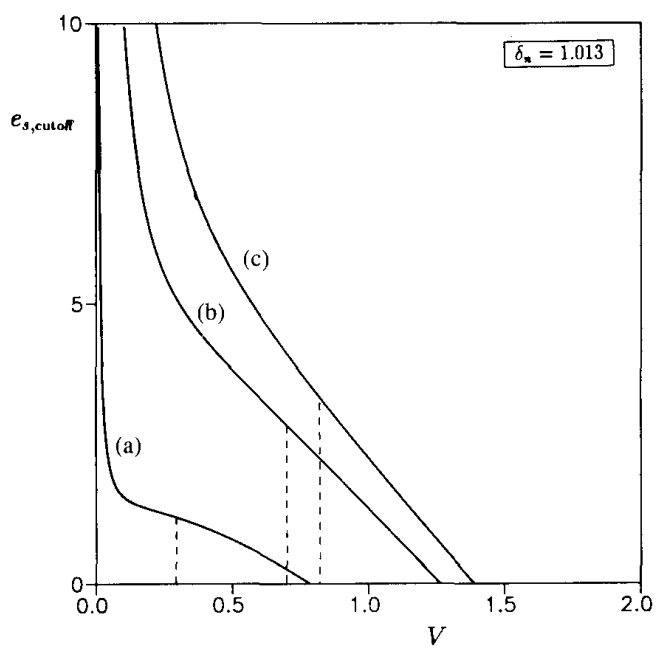

Fig. 11. Normalized cutoff power $\left(\mathrm{TE}_{0}\right)$ as a function of the normalized thickness, for different values of the asymmetry measure: (a) $a=1$, (b) $a$ $=10$, and (c) $a=30$. Self-focusing bounding medium. Dashes indicate the value of $V_{c \text { c }}$ corresponding to the different cases. Above $V_{c s}$ cutoff takes place through a bulged guided wave and when $V<V_{c s}$ it occurs through a surface wave.

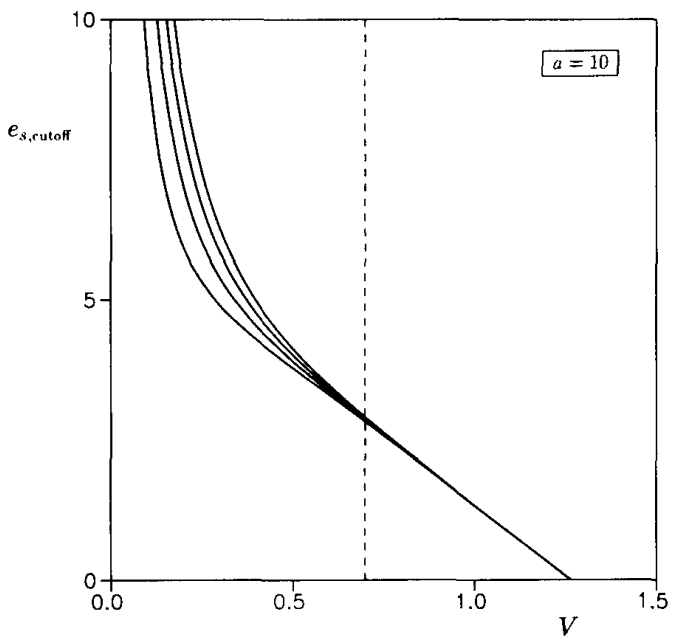

Fig. 12. Normalized cutoff power $\left(\mathrm{TE}_{0}\right)$ versus the normalized thickness in an asymmetric waveguide with a self-focusing substrate, for different values of the parameter $\delta_{n}$. From left to right: $\delta_{n}=1.01,1.02,1.03$, and 1.04. Dashes indicate the value of $V_{c s}$.

tively. As expected from Fig. 2, for a given value of the normalized thickness, the threshold value of $e_{s}$ that ensures guided propagation grows as the asymmetry measure of the waveguide increases. In the range $V_{L \text {, cutoff }}>$ $V>V_{c s}$, cutoff occurs through a bulged guided wave, whereas when $V<V_{c s}$ it takes place through a surface wave. When the normalized thickness approaches $V=0$, $e_{s, \text { cutoff }}$ grows very quickly, but for $V_{L, \text { cutoff }}>V>V_{c s}$ it amounts to relatively small values. On the other hand, Fig. 12 shows that in an asymmetric waveguide, for a given value of the normalized thickness, $e_{s, \text { cutoff }}$ increases with $\delta_{n}$, although at a slow rate. This behavior agrees with the plots in Fig. 8 and comes from the fact that when $\delta_{n}$ increases the self-focusing effect at the substrate must be more and more important in order that there be an allowed guided solution below the low-power cutoff thickness. Finally, according to the expression of $e_{s \text {, cutoff }}$ obtained for values of $V$ near $V_{L, \text { cutoff }}$, the plots in Fig. 12 corresponding to different values of $\delta_{n}$ practically coincide in this range.

In the case of a self-defocusing substrate, the cutoff occurs when $b \rightarrow 0$. Taking this limit into the corresponding eigenvalue equation, the critical maximum value of $\left|e_{s}\right|$ in order that guided propagation occurs is found to be

$$
\begin{aligned}
\left|e_{s, \text { cutoff }}\right|= & 2 V \frac{\tan (V)-\sqrt{a}}{1+\sqrt{a} \tan (V)} \\
& +2 V^{2} \frac{[\sin (V)-\sqrt{a} \cos (V)]^{2}}{[\cos (V)+\sqrt{a} \sin (V)]^{4}} \\
& +\left\{\frac{1}{2 V \sqrt{a}}+\frac{1+a}{2}+\frac{1-a}{2} \frac{\sin (2 V)}{2 V}\right. \\
& \left.+\sqrt{a} \frac{\sin ^{2}(V)}{V}\right\} .
\end{aligned}
$$

In contrast to (39), this expression applies in the range $V_{c d}$ $>V \geq V_{L \text {, cutoff. The upper limit for } V \text { is due to the fact }}$ that when the normalized thickness is greater than $V_{c d}$, there is not critical power. In fact, it is easy to verify that when $b \rightarrow b_{d 0}$, the expression of $I_{s}$ corresponding to the self-defocusing case diverges. This fact indicates that for given values of the asymmetry measure and the parameters $\delta_{n}$, when $\left|e_{s}\right| \rightarrow \infty$, the curve $b\left(V ; e_{s}\right)$ approaches asymptotically to the curve $b_{d 0}(V)$ defined by (37). We must also note that (40) makes no sense in the case of a symmetric $(a=0)$ waveguide. This is so because, as has been pointed out in [26], in the case of $a=0$, when the guided wave reaches its cutoff, the guided power diverges. The behavior of $\left|e_{s, \text { cutoff }}\right|$ as a function of $V$ is shown in Fig. 13 for different values of $a$. Since $\left|e_{s \text {, cutoff }}\right|$ no longer depends on $\delta_{n}$, this is a universal plot.

\section{Concluding Remarks}

In previous sections we have shown that normalized variables provide a powerful tool for the description of the waveguiding properties of a slab waveguide with a nonlinear Kerr-like substrate or cladding. We have demonstrated that the stationary waveguiding properties of TE waves guided by such a structure can be completely described in terms of one dependent $(b)$ and four independent parameters $\left(V, a, e_{s}\right.$, and $\left.\delta_{n}\right), \delta_{n}$ playing only a relevant role in some special situations. These variables enable us to identify in a very simple way the similarity rules existing between different waveguides and the obtaining of universal results showing the above-mentioned waveguiding properties by means of a few plots. In addition, $V, a, e_{s}$, and $\delta_{n}$ can be directly related to the actual parameters of the waveguide and thus with the design variables. 


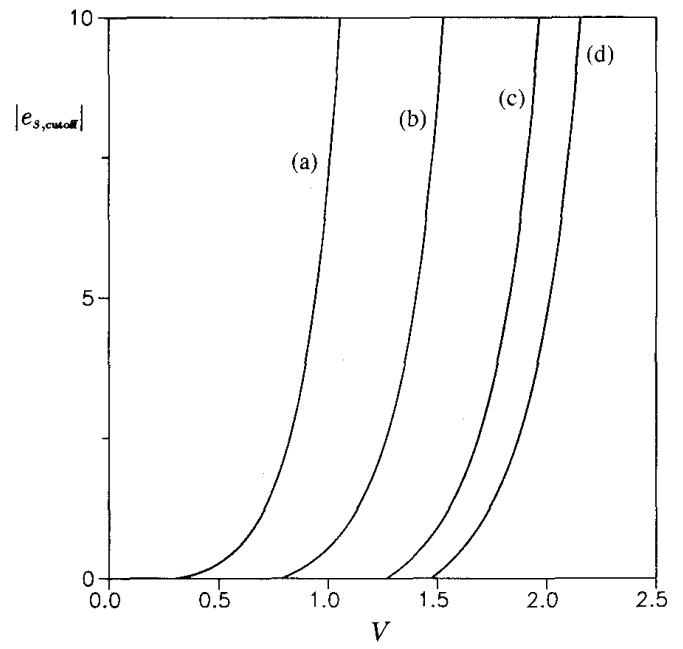

Fig. 13. Normalized cutoff power $\left(\mathrm{TE}_{0}\right)$ as a function of the normalized thickness, for different values of the asymmetry measure: (a) $a=0.1$, (b) $a=1$, (c) $a=10$, and (d) $a=100$. Self-defocusing bounding medium.

In the $b-V$ charts, allowed and forbidden bands in order to stationary guided propagation occurs have been recognized and classified, with the marginal loci separating different regions being a function of only the parameters $V$ and $a$. This makes possible to partition the parameter space into regions of similar behavior, in such a way that the number and type of possible nonlinear stationary waves guided by a particular waveguide can be read off inmediately from the corresponding $b-V$ diagram.

This fact has interesting implications. For instance, we conclude that, in the case of a self-focusing substrate, the value of the normalized thickness $V$ determines by itself the different types of possible guided solutions which are allowed in a particular waveguide. If $V>V_{L \text {, cutoff }}$, all surface wave, bulged guided wave and pure guided wave solutions are possible; when $V_{L \text {, cutoff }}>V>V_{c s}$, only surface wave and bulged guided wave solutions can exist in the waveguide; finally, when $V<V_{c s}$, both bulged and pure guided wave solutions become no longer allowed and only surface wave solutions remain possible. We must also emphasize that the critical value $V_{c s}$, like $V_{L \text {, cutoff }}$, has been found to depend only on the asymmetry measure of the waveguide and vanishes in the case of a perfectly symmetric structure $(a=0)$.

When considering self-defocusing materials, another $a$-dependent critical normalized thickness arises, $V_{c d}$. Below it, $V_{c d}>V>V_{L \text {, cutoff, there is a maximum allowed }}$ guided power, but when $V>V_{c d}$ the guided wave power can take any value. Thus, $V_{c d}$ separates the region in the parameter space where the nonlinear waveguide can act as a power limiter from the region where it cannot.

We have obtained the analytic expressions of the marginal loci separating the different allowed from the forbidden regions in the $b-V$ plane. The knowledge of these critical values, $b_{c+}, b_{c-}, b_{c 0}$, and $b_{d 0}$, has enabled us to calculate the expression of the normalized cutoff power (threshold power in the case of self-focusing media and maximum power for self-defocusing media) in order to guided propagation occurs, as a function of the waveguide parameters.

Here we have mainly limited ourselves to the lowest order TE solution, but the analysis of higher-order $\mathrm{TE}_{m}$ guided waves goes as well. Likewise, in this paper we have addressed the case of a waveguide with a nonlinear bounding medium, but the approach used is intended to be more general. For instance, the reported set of normalized variables hold as well in the case of a structure with a nonlinear film, provided that film parameters are used in the definition of the normalized power measure (to differentiate both cases now it will be denoted as $e_{f}$ ). According to (17) one has

$$
e_{f}=2 n_{f} \Pi_{1}^{2} \frac{n_{2 f} P}{D}
$$

and $b=B_{f}\left(V, a, e_{f}, \delta_{n}\right)$. The reported normalized parameters can be used also when both substrate and film (or substrate and cover) materials are nonlinear. In this case the additional generalized aspect ratio

$$
\delta_{\alpha}=\frac{n_{2 f}}{n_{2 s}}\left(\text { or } \delta_{\alpha}=\frac{n_{2 c}}{n_{2 s}}\right)
$$

will only be required.

Similarity rules existing between waveguides with other than Kerr-like nonlinearities may be investigated by the proposed procedure. This seems to be a very attractive goal, since in most cases for such nonlinearities an analytical solution of the nonlinear wave equation is not available and thus the use of general numerical methods is needed [27]-[30]. Saturable nonlinearities are good candidates since results similar to those obtained for the Kerr-like case are expected.

Finally, similarity rules can provide a potential tool for the study of stability of nonlinear guided modes. This is complicated subject and to date the analysis is usually performed by means of numerical methods (the beam propagation method or solution of the nonlinear Schrödinger equation using numerical techniques) involving to launch the stationary solutions into the waveguide. This approach require a great volume of calculations, so an exhaustive analysis of the stability of a particular class of nonlinear guided waves require a heavy computational effort. This fact suggests that stability analysis would greatly benefit if the tested stationary solutions could be expressed in terms of a minimal number of independent parameters, as has been done for the particular case treated here, so that the degrees of freedom of the problem become as small as possible. This statement is particularly true in order to obtain the locus of marginal stability (separating stable from unstable solutions) in the waveguide parameter space, which is the final goal of the stability analysis. 


\section{REFERENCES}

[1] N. N. Akhmediev, "Novel class of nonlinear surface waves: Asymmetric modes in a symmetric layered structure," Sov. Phys. JETP, vol. 56, pp. 299-303, 1982

12] F. Lederer, U. Langbein, and H.-E. Ponath, "Nonlinear waves guided by a dielectric slab," Appl. Phys. B, vol. 31, pp. 69-73, 1983.

(3) U. Langbein, F. Lederer, H.-E. Ponath, and U. Trutschsel, "Dispersion relations for nonlinear guided waves," J. Molec. Struc., vol. 115. pp. 493-496, 1984.

[4] D. Mihalache, D. Mazilu, and H. Totia, "Bistable states of s-polarized nonlinear waves guided by an asymmetric three-layer dielectric structure," Phys. Scr., vol. 30, pp. 335-340, 1984.

[5] A. D. Boardman and P. Egan, "S-polarized waves in a thin dielectric film asymmetrically bounded by optically nonlinear media," IEEE $J$. Quantum Electron., vol. QE-21, pp. 1701-1713, 1985

16] G. I. Stegeman and C. T. Seaton, "Nonlinear integrated optics," $J$. Appl. Phys., vol. 58, pp. R57-78, 1985.

[7] G. I. Stegeman, E. M. Wright, N. Finlayson, R. Zanoni, and C. T. Seaton. "Third order nonlinear integrated optics," J. Lightwave Technol., vol. 6, pp. 953-970, 1988.

[8] D. Mihalache, D. Mazilu, M. Bertolotti, and C. Sibilia, "Exact solution for nonlinear thin-film guided waves in higher-order nonlinear media," J. Opt. Soc. Amer. B, vol. 5, pp. 565-570, 1988.

[9] G. I. Stegeman, E. M. Wright, C. T. Seaton, J. V. Moloney, T.-P. Shen, A. A. Maradudin, and R. F. Wallis, "Nonlinear slab-guided waves in non-Kerr-like media," IEEE J. Quantum Electron., vol. QE-22, pp. 977-983, 1986.

[10] U. Langbein, F. Lederer, T. Peschel, and H.-E. Ponath, "Nonlinea guided waves in saturable nonlinear media," Opt. Lett., vol. 10, pp. $571-573,1985$.

[11] S. J. Al-Bader and H. A. Jamid, "Nonlinear waves in saturable selffocusing thin films bounded by linear media," IEEE J. Quantum Electron., vol. 24, pp. 2052-2058, 1988

[12] J. B. J. Fourier, Théorie Analytique de la Chaleur, Paris, 1822, English translation, The Analytical Theory of Heat. Cambridge: Freeman, 1878 .

[13] E. Buckingham, "On physically similar systems; illustrations of the use of dimensional equations," Phys. Rev., vol. 4, pp. 345-376, 1914.

[14] P. W. Bridgman, Dimensional Analysis. New Haven, CT: Yale Univ. Press, 1931.

[15] H. Kogelnik and V. Ramaswamy, "Scaling rules for thin-film optical waveguides," Appl. Opt., vol. 13, pp. 1857-1862, 1974.

[16] G. B. Hocker and W. K. Burns, "Modes in diffused optical waveguides of arbitrary index profiles," IEEE J. Quantum Electron., vol. QE-I1, pp. 270-276, 1975.
[17] L. Riviere, A. Yi-Yan, and H. Carru, "Properties of single-mode planar waveguides with a gaussian profile,"'J. Lightwave Technl., vol. LT-3, pp. $368-377,1985$

[18] L. Torner, F. Canal, and J. Hernández-Marco, "Cutoff behavior of graded-index slab waveguides," Opt. Quantum Electron., vol. 21, pp. 451-462, 1989

[19] G. A. Bennett and C.-L. Chen, "Wavelength dispersion of optical waveguides," Appl. Opt., vol. 19, pp. 1990-1995, 1980.

[20] Y.-F. Li and J. W. Y. Lit, "General formulas for the guiding properties of a multilayer slab waveguide," J. Opt. Soc. Amer. A, vol. 4, pp. 671-677, 1987.

[21] N. Dagli and C. G. Fonstad, "Universal design curves for tib waveguides," J. Lightwave Technol., vol. 6, pp. 1136-1145, 1988.

[22] J. Recolons, L. Torner, and F. Canal, "Normalized parameters for Y-branch optical waveguides," Opt. Lett., vol. 16, pp. 636-638, 1991.

[23] S. Chelkowski and J. Chrostowski, "Scaling rules for slab waveguides with nonlinear substrate," Appl. Opt., vol. 26, pp. 3681-3686, 1987.

[24] M. Fontaine, "Scaling rules for nonlinear thin film optical waveguides," Appl. Opt., vol. 29, pp. 3891-3899, 1990.

[25] A. E. Kaplan, "Theoy of hysteresis reflection and refraction of light by a boundary of a nonlinear medium," Sov. Phys. JETP, vol. 45 , pp. 896-905, 1977.

[26] C. T. Seaton, J. D. Valera, R. L. Shoemaker, G. I. Stegeman, J. T. Chilwell, and S. D. Smith, "Calculations of nonlinear TE waves guided by thin dielectric films bounded by nonlinear media," IEEE J. Quantum Electron., vol. QE-21, pp. 774-783, 1985.

[27] F. Dios, L. Torner, and F. Canal, "Self-consistent solution for general nonlinear slab waveguides," Opt. Commun., vol. 72, pp. 54-59, 1989

[28] M. R. Ramadas, R. K. Varshney, K. Thyagarakam. amd A. K. Ghatak, "A matrix approach to study the propagation characteristics of general nonlinear planar waveguide,' J. Lightwave Technol., vol. 7 , pp. 1901-1905, 1989.

[29] K. Hayata, M. Nagai, and M. Koshiba, "Finite-element formalism for nonlinear slab-guided waves," IEEE Trans. Microwave Theory Tech., vol. 36, pp. 1207-1215, 1988.

[30] K. Ogusu, "Computer analysis of general nonlinear planar waveguides," Opt. Commun., vol. 64, pp. 425-430, 1987.

Lluís Torner, photograph and biography not available at the time of publication.

Juan P. Torres, photograph and biography not available at the time of publication 